\title{
Numerical Simulation of Charged Fullerene Spectrum
}

\section{Rafael Arutyunyan, Yuri Obukhov and Petr Vabishchevich}

\author{
Nuclear Safety Institute, Russian Academy of Sciences \\ B. Tulskaya 52, 115191 Moscow, Russia \\ E-mail: arut@ibrae.ac.ru \\ E-mail: obukhov@ibrae.ac.ru \\ E-mail(corresp.): vab@ibrae.ac
}

Received August 3, 2018; revised February 16, 2019; accepted February 18, 2019

\begin{abstract}
The mathematical model of the ground state electron spectrum of a charged fullerene is constructed on the basis of the potential of a charged sphere and the spherically symmetric potential of a neutral fullerene, derived in a singleelectron self-consistent field model approach. The electron spectrum is defined as the solution of the spectral problem for the one-dimensional Schrödinger equation. For the numerical solution of the spectral problem, piecewise-linear finite elements are used. The computational algorithm was tested on the analytical solution of the problem of the spectrum of the hydrogen atom. For solution of matrix spectral problems, a free library for solving spectral problems of SLEPc is used. The results of calculations of the electron spectrum of a charged fullerene $C_{60}$ are presented.
\end{abstract}

Keywords: fullerene, Schrödinger equation, spectral problem, finite element method.

AMS Subject Classification: 65L15; 65L60; 65Z05.

\section{Introduction}

Fullerenes are known as one of allotropes of carbon, along with nanotubes, graphite, diamond, graphene and amorphous carbon. The first fullerene $C_{60}$ molecule was experimentally discovered in the 1980-ies using earlier theoretical predictions $[18,19]$. It represents a nanometer-size hollow spherical structure consisting of 60 carbon atoms, which are located at the vertices of a truncated icosahedron. Later, the fullerene nanotubes and many other fullerenes were

Copyright (c) 2019 The Author(s). Published by VGTU Press

This is an Open Access article distributed under the terms of the Creative Commons Attribution License (http://creativecommons.org/licenses/by/4.0/), which permits unrestricted use, distribution, and reproduction in any medium, provided the original author and source are credited. 
discovered, such as $C_{20}, C_{70}$ and even larger structures due to started production of fullerenes in large quantities. This gave a birth to an incredible growth of research in the area of nanoscience, the historic development and the current status of which can be found in $[9,21,35]$.

In past decades, the properties of charged fullerenes have been actively investigated, both experimentally and theoretically $[12,25,26,32,34,38,41,42,43]$. A large amount of works are devoted to the study of their stability (lifetime), mechanisms for their charging and decay [35, Ch.25].

In our article [3] the structure of the electronic spectrum of the charged fullerenes is investigated numerically. Simple models are used to show the existence of the volume-localized discrete quantum levels for the usual fullerene. The current paper should be considered as supplementary to the recent publication [3] which provides a general introduction to the subject. With an account of the well-known fact that geometrically the shape of a $C_{60}$ fullerene (which is a truncated icosahedron with carbon atoms located in its 60 equivalent vertices) is very close to a sphere, it is common to study the electronic structure of fullerenes (and, in general, interaction of electrons and photons with fullerenes and fullerene-like systems) by means of the simple model potentials. It is worthwhile to mention the use of the square-well type potentials $[10,11,16,40]$, of the Dirac bubble and Gaussian-type potentials [1,13,24], as well as self-consistent models for the fullerene cage derived from a spherical jellium shell $[25,29,36]$. Here we focus on the computational issues of the numeric modelling, on the analysis of the corresponding discrete problem, and on the numerical solution of actual spectral problems with an account of peculiar features of the physical system under consideration. Furthermore, the conclusions of [3] are checked here by making use of the different values for the Lorentz-bubble potential.

A spectral problem is solved for the one-dimensional spherically symmetric Schrödinger equation with the corresponding potential [20]. Numerical algorithm taking into account the features of the problem is based on finiteelement approximation [8]. To solve the corresponding matrix spectral problem we use the SLEPc (Scalable Library for Eigenvalue Problem Computations, http://slepc.upv.es/) [14]. We use a Krylov-Schur algorithm, a variation of Arnoldi method, proposed in [37]. The development of the computational algorithm for solving the spectral problem was carried out on the test problem of the hydrogen atom spectrum, which has an exact analytical solution.

\section{Problem statement}

For modeling of the electronic spectrum of the charged fullerenes spherical symmetry approximations are used. Here we confine our attention to the case of the positively charged fullerenes. In practice, one can multiply ionize $C_{60}$ with the help of the highly charged ions, fast electrons, or photons [9]. Experimentally, charged fullerenes in the range of $Z=0, \cdots, 9 e,[17]$, and even up to $Z=10 e,[6,7]$. Basic notations are as follows: $m_{e}$ and $e$ are electron's mass and the absolute value of electron charge, $\varepsilon_{0}$ is the electric constant of vacuum, $\hbar$ is Planck constant, $a_{0}=4 \pi \varepsilon_{0} \hbar^{2}\left(m_{e} e^{2}\right)^{-1}$ is the Bohr radius.

Let us formulate the corresponding quantum-mechanical spectral problem. 
With an account of the spherical symmetry of a fullerene, for the wave function we use the standard ansatz $\psi(r, \vartheta, \varphi)=R(r) Y_{l m}(\vartheta, \varphi)$. Here $R(r)$ is the radial part of the wave function is determined in the self-consistent potential of the fullerene ion, $Y_{l m}(\vartheta, \varphi)$ is the spherical harmonic, $l$ is the quantum number of angular momentum, $m$ is the quantum number of its $z$-projection. The spherically symmetric Schrödinger equation is written [20] into a second order differential equation

$$
\frac{d^{2} \chi}{d r^{2}}-\frac{l(l+1)}{r^{2}} \chi+\frac{2 m_{e}}{\hbar^{2}}(E-U(r)) \chi=0
$$

for the function $\chi(r)=r R(r), 0 \leq r<\infty$, under the boundary conditions

$$
\chi(0)=0, \quad \chi(\infty)=0 .
$$

The problem consists in finding electron energy levels $E$ for a given potential $U(r)$.

For numerical solution of the spectral problem (2.1)-(2.2) we use the atomic system of units, in which $m_{e}=|e|=\hbar=1$, and $a_{0}$ denotes the length scale. The equation (2.1) in this case is written in the form

$$
\frac{d^{2} \chi}{d r^{2}}-\frac{l(l+1)}{r^{2}} \chi+2(E-U(r)) \chi=0 .
$$

We will discuss the energy levels of an electron by starting from a simple model potential, and then move on to more complicate form of $U$. In the simplest model, one can describe a charged fullerene by the potential of a sphere with a constant surface charge density:

$$
U_{1}(r)=-Z\left\{\begin{array}{cc}
1 / R, & r \leq R, \\
1 / r, & r>R,
\end{array}\right.
$$

where $Z=N e$ is a positive charge, and $R$ is the fullerene radius. Our attention will be mainly confined to the $C_{60}$ fullerene, when $R=6.665$.

The model above provides a rather simplified description in the sense that it does not take into account the actual physical structure of a fullerene. A more realistic single-electron potential $U(r)$ can be constructed in the framework of the jellium model $[4,5,15]$ as a sum of the positive contribution of the carbon atom's nuclei located on the spherical surface of the fullerene radius $R$ and the negative contribution of the electron clouds. The resulting potential is attractive and it has a cusp-shape form with the clear localization in the thin spherical shell. For $C_{60}$, the corresponding Lorentz-bubble potential reads

$$
U_{2}(r)=-\frac{V}{(r-R)^{2}+d^{2}},
$$

where the parameter $V$ determines the depth, $d$ - the width, and $R$ - the position.

Although the electronic structure of fullerenes is widely studied $[1,10,11,13$, $16,24,29,36]$ in the framework of the simple model potentials approach, such 
investigations have obvious limitations. More nontrivial shape of potential can be derived from the photoelectron intensity measurements for $C_{60}$ fullerenes $[25,30]$. The spectral computations were also done by taking into account of the actual fullerene's nonspherical geometry $[33,39]$ for electrically neutral systems, and for the charged fullerenes the corresponding DFT computations [2] yield the qualitatively consistent results.

\section{Computational algorithm}

The radial Schrödinger problem (2.2)-(2.3) is the example of self-adjoint SturmLiouville problems [44]. To find eigenvalues and eigenfunctions, we can use various numerical methods [27]. Developed specialized software for these tasks (see, for example, $[22,28]$ ) is widely used.

In our study, we focus on the use of standard approximations by finite elements [8] and general computational algorithms for solving spectral problems for partial differential equations, which are based on the technology of working with sparse matrices [31].

The spectral problem (2.2)-(2.3) has singularities that must be taken into account when solving it numerically. The first of these is related to the singularity

$$
q(r)=\frac{l(l+1)}{r^{2}}
$$

at $r=0$. We used the simplest approach, when $q(r)$ is replaced by

$$
q_{\varepsilon}(r)=\frac{l(l+1)}{r^{2}+\varepsilon}
$$

with a sufficiently small value $\varepsilon$. In addition, the problem is considered on a bounded interval $[0, \bar{r}]$ by choosing a sufficiently large value $\bar{r}$. Thus, we seek a solution of equation

$$
\frac{d^{2} \chi}{d r^{2}}-\frac{l(l+1)}{r^{2}+\varepsilon} \chi+2(E-U(r)) \chi=0
$$

under the boundary conditions

$$
\chi(0)=0, \quad \chi(\bar{r})=0 .
$$

An approximate solution of the problem (3.1)-(3.2) is searched for using a uniform grid:

$$
r_{k}=k h, \quad k=0,1, \ldots, M, \quad M h=\bar{r} .
$$

When using Lagrange finite elements of the first order (piece-wise linear approximation), we can define the hat function $\varphi_{k}(r) \subset V^{h}, k=1,2, \ldots, M-1$, where

$$
\varphi_{k}\left(r_{j}\right)= \begin{cases}1, & \text { if } k=j, \\ 0, & \text { if } k \neq j .\end{cases}
$$

For $v \in V_{h}$, we have

$$
v(r)=\sum_{k=1}^{M-1} v_{k} \varphi_{k}(r),
$$


where $v_{k}=v\left(r_{k}\right), k=1,2, \ldots, M-1$.

The pair $(u, E)$ (the eigenfunction $u \in V_{h}$ and the eigenvalue $E$ ) is determined from the following conditions

$$
a(u, v)=E b(u, v), \quad \forall v \in V_{h} .
$$

For bilinear forms we have

$$
\begin{aligned}
& a(u, v)=\int_{0}^{\bar{r}} \frac{d u}{d r} \frac{d v}{d r} d r+\int_{0}^{\bar{r}}\left(\frac{l(l+1)}{r^{2}+\varepsilon}+2 U(r)\right) u v d r \\
& b(u, v)=2 \int_{0}^{\bar{r}} u v d r
\end{aligned}
$$

The problem (3.2) is associated with the matrix eigenvalue problem

$$
A \boldsymbol{u}=E B \boldsymbol{u}, \quad \boldsymbol{u}=\left(u_{1}, u_{2}, \ldots, u_{M-1}\right) .
$$

Here $A=\left(A_{k j}\right)$ is matrix with elements $a_{k j}=a\left(\varphi_{k}, \varphi_{j}\right)$ and $B=\left(B_{k j}\right)$, $b_{k j}=b\left(\varphi_{k}, \varphi_{j}\right)$.

To find the first eigenvalues and eigenfunctions of the problem (3.3) with sparse matrices $A$ and $B$, one can use various numerical methods [31]. We apply Krylov-Schur algorithm [37], which is a variant of the Arnoldi method. The software implementation is performed on the FEniCS [23] computational platform. Spectral problems are solved using the library SLEPc [14].

The computational algorithm and the corresponding software thus developed was tested on the well-known problem for the hydrogen atom. In this case the potential has the form

$$
U(r)=-1 / r .
$$

The exact solution of the problem (2.2), (2.3), (3.4) is well known - see, for example, [20]. The energy levels have the form

$$
E=-\frac{1}{2} \frac{1}{(l+i)^{2}}, \quad l=0,1, \ldots, \quad i=1,2, \ldots,
$$

where $i=m+1$ is the eigenvalue number for a given $l$.

The numerical solution of the spectral problem (3.1)-(3.2) with the potential (3.3) is problematic because of the singularity of the functional for $r \rightarrow 0$. Therefore, instead of (3.4) we use the approximate functional

$$
\tilde{U}(r)=\left\{\begin{array}{cc}
-1 / r_{0}, & r \leq r_{0}, \\
-1 / r, & r>r_{0},
\end{array}\right.
$$

with a sufficiently small $r_{0}$.

The results of calculations for values of $r_{0}=0.05$ and $r_{0}=0.1$ in (3.6) are presented in the Table 1 with the parameter $\varepsilon=1 \cdot 10^{-5}$. This value is fixed from the methodical computations and it does not affect the precision for the approximate solution of spectral problems under consideration. The data for $\bar{r}=200$ are given for $M=4000$, the exact value is determined according to 
(3.5). There is good accuracy in calculation of the energy level. The major error is related to the ground state eigenvalue. Using a more detailed grid (see the results for the $M=5000$ case) makes it possible to increase the accuracy of calculating of the ground state eigenvalue. The computations are done on such fine grids in order to obtain the three-digit precision for the eigenvalues.

Table 1. Energy levels (hydrogen atom)

\begin{tabular}{ccccccc}
\hline$n$ & $l$ & $i$ & $r_{0}=0.05$ & $r_{0}=0.1$ & $r_{0}=0.05(N=5000)$ & exact \\
\hline 1 & 0 & 1 & -0.50109 & -0.49596 & -0.49950 & -0.50000 \\
2 & 0 & 2 & -0.12510 & -0.12496 & -0.12497 & -0.12500 \\
3 & 1 & 1 & -0.12496 & -0.12446 & -0.12492 & -0.12500 \\
4 & 0 & 3 & -0.05558 & -0.05555 & -0.05555 & -0.05556 \\
5 & 2 & 1 & -0.05555 & -0.05554 & -0.05555 & -0.05556 \\
6 & 1 & 2 & -0.05554 & -0.05539 & -0.05553 & -0.05556 \\
7 & 0 & 4 & -0.03126 & -0.03125 & -0.03125 & -0.03125 \\
8 & 3 & 1 & -0.03125 & -0.03125 & -0.03125 & -0.03125 \\
9 & 2 & 2 & -0.03125 & -0.03124 & -0.03125 & -0.03125 \\
10 & 1 & 3 & -0.03124 & -0.03118 & -0.03124 & -0.03125 \\
11 & 0 & 5 & -0.02001 & -0.02000 & -0.02000 & -0.02000 \\
12 & 4 & 1 & -0.02000 & -0.02000 & -0.02000 & -0.02000 \\
13 & 3 & 2 & -0.02000 & -0.02000 & -0.02000 & -0.02000 \\
14 & 2 & 3 & -0.02000 & -0.02000 & -0.02000 & -0.02000 \\
15 & 1 & 4 & -0.02000 & -0.01996 & -0.01999 & -0.02000 \\
16 & 0 & 6 & -0.01389 & -0.01389 & -0.01389 & -0.01389 \\
17 & 5 & 1 & -0.01389 & -0.01389 & -0.01389 & -0.01389 \\
18 & 4 & 2 & -0.01389 & -0.01389 & -0.01389 & -0.01389 \\
19 & 3 & 3 & -0.01389 & -0.01389 & -0.01389 & -0.01389 \\
20 & 2 & 4 & -0.01389 & -0.01389 & -0.01389 & -0.01389 \\
\hline
\end{tabular}

The matrix spectral problem does not completely inherit the properties of the differential problem. In particular, the computed levels 4-6 and so on are not degenerate. The graphical representation of the spectrum at $r_{0}=$ 0.05 is given in Figure 1(a). The corresponding eigenfunctions are shown in Figure 1(b). The following normalization of eigenfunctions is used

$$
\|R\|=1, \quad\|R\|^{2}=4 \pi \int_{0}^{\infty} r^{2} R^{2}(r) d r .
$$

\section{Results of calculations}

First, we present the calculated data on the electron spectrum of an neutral fullerene $C_{60}$, when $U(r)=U_{2}(r)$. Following [4], we studied in [3] the case

$$
V=0.711, \quad R=6.665, \quad d=0.610 \text {. }
$$

However, the Lorentz-bubble potential with these values of parameters does not yield the correct affinity energy for $C_{60}$. Here we take the more accurate values which provide the agreement with the detachment energy $2.65 \mathrm{eV}$ found experimentally from the photoelectron spectroscopy. Namely, we use the potential 


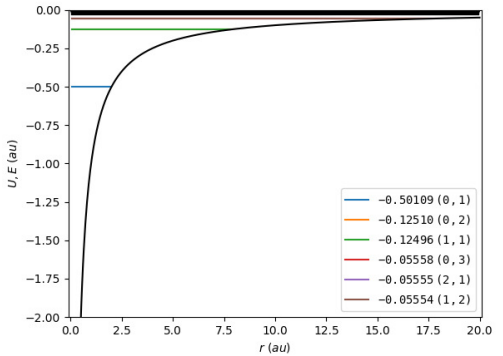

a)

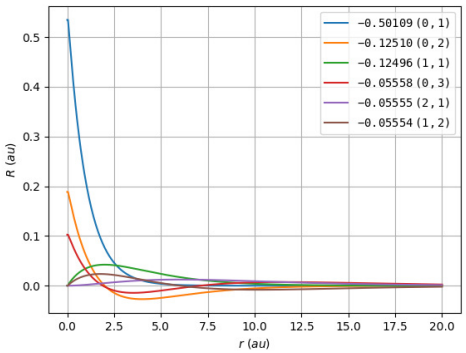

b)

Figure 1. Results for the hydrogen atom: a) numeric evaluation of the spectrum, b) radial wave functions $R(r)$ for the lowest energy levels.

$(2.5)$, where

$$
V=0.1104, \quad R=6.627, \quad d=0.5 .
$$

Calculated data are given in Table 2 and in Figure 2. Numeric computations of the spectrum for different fullerene models are done on the grid with $M=4000$.

Table 2. Lowest energy levels for neutral fullerene in the potential (2.5) with $V=$ $0.1104, \quad R=6.627, \quad d=0.5$

\begin{tabular}{cccc}
\hline$n$ & $l$ & $i$ & $E$ \\
\hline 1 & 0 & 1 & -0.09741 \\
2 & 1 & 1 & -0.07123 \\
3 & 2 & 1 & -0.02595 \\
\hline
\end{tabular}

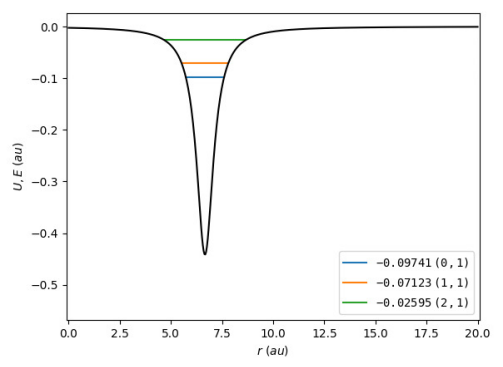

a)

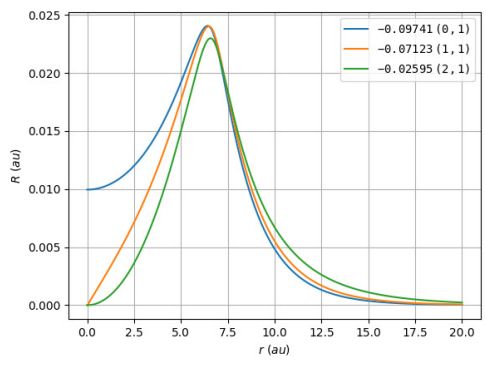

b)

Figure 2. Results for neutral fullerene with (2.5): a) the spectrum structure, b) radial wave function $R(r)$.

For the case of the charged sphere model, we set $U(r)=U_{1}(r)$, where $U_{1}(r)$ is defined for different values of $Z$ according to (2.4). Numeric results for the 
spectrum with $Z=1$ are presented in Table 3 and in Figure 3. Analogous data for the case $Z=5$ is given in Table 4 and in Figures 4 .

Table 3. The energy levels for the potential (2.4) a charged sphere with $Z=1$

\begin{tabular}{cccccccc}
\hline$n$ & $l$ & $i$ & $E$ & $n$ & $l$ & $i$ & $E$ \\
\hline 1 & 0 & 1 & -0.11245 & 7 & 3 & 1 & -0.03109 \\
2 & 1 & 1 & -0.08060 & 8 & 2 & 2 & -0.02909 \\
3 & 0 & 2 & -0.05497 & 9 & 1 & 3 & -0.02465 \\
4 & 2 & 1 & -0.05134 & 10 & 0 & 4 & -0.02036 \\
5 & 1 & 2 & -0.04076 & 11 & 4 & 1 & -0.02000 \\
6 & 0 & 3 & -0.03165 & 12 & 3 & 2 & -0.01988 \\
\hline
\end{tabular}

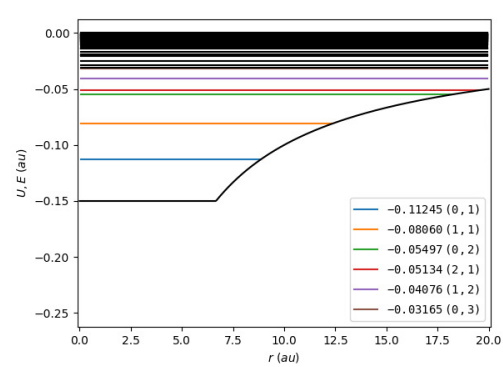

a)

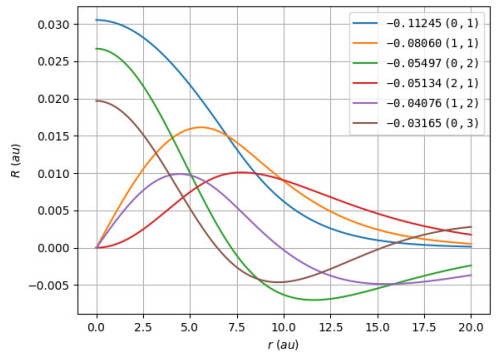

b)

Figure 3. Results for the potential (2.4) of charged sphere with $Z=1$ : a) the spectrum, b) radial wave functions $R(r)$ for the lowest levels.

Table 4. The energy levels for the potential (2.4) of a charged sphere with $Z=5$

\begin{tabular}{cccccccc}
\hline$n$ & $l$ & $i$ & $E$ & $n$ & $l$ & $i$ & $E$ \\
\hline 1 & 0 & 1 & -0.69161 & 7 & 4 & 1 & -0.40274 \\
2 & 1 & 1 & -0.63311 & 8 & 0 & 3 & -0.39074 \\
3 & 2 & 1 & -0.56307 & 9 & 2 & 2 & -0.39010 \\
4 & 0 & 2 & -0.53875 & 10 & 1 & 3 & -0.33711 \\
5 & 3 & 1 & -0.48487 & 11 & 3 & 2 & -0.33262 \\
6 & 1 & 2 & -0.45857 & 12 & 5 & 1 & -0.32240 \\
\hline
\end{tabular}

For a charged fullerene, we set $U(r)=U_{1}(r)+U_{2}(r)$. The results of calculations for $Z=1$ are presented in Table 5 and in Figure 5 . The effect of increasing of the charge of fullerene is traced on the calculated data for the case $Z=5$. The first 12 energy levels are presented in the Table 6 , the spectrum and basic eigenfunctions are shown in Figure 6 . 


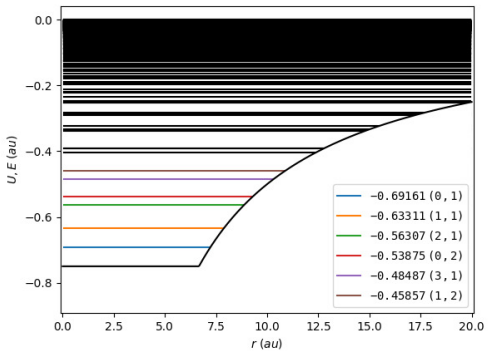

a)

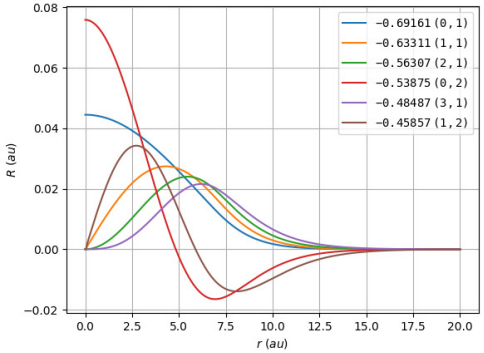

b)

Figure 4. Results for the potential (2.4) of charged sphere with $Z=5$ : a) the spectrum, b) radial wave functions $R(r)$ for the lowest levels.

Table 5. The energy levels of a charged fullerene in the potential $U(r)=U_{1}(r)+U_{2}(r)$ for $Z=1$

\begin{tabular}{cccccccc}
\hline$n$ & $l$ & $i$ & $E$ & $n$ & $l$ & $i$ & $E$ \\
\hline 1 & 0 & 1 & -0.23745 & 7 & 2 & 2 & -0.03989 \\
2 & 1 & 1 & -0.20944 & 8 & 0 & 3 & -0.03604 \\
3 & 2 & 1 & -0.16068 & 9 & 3 & 2 & -0.02868 \\
4 & 3 & 1 & -0.09567 & 10 & 1 & 3 & -0.02834 \\
5 & 0 & 2 & -0.06295 & 11 & 4 & 1 & -0.02545 \\
6 & 1 & 2 & -0.05092 & 12 & 2 & 3 & -0.02341 \\
\hline
\end{tabular}

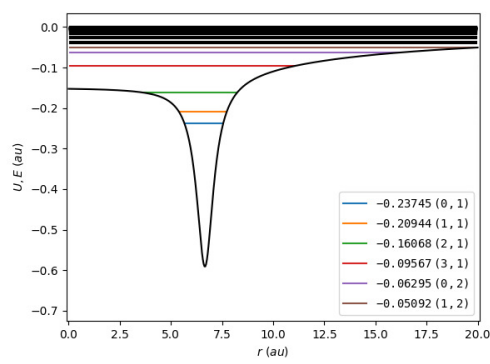

a)

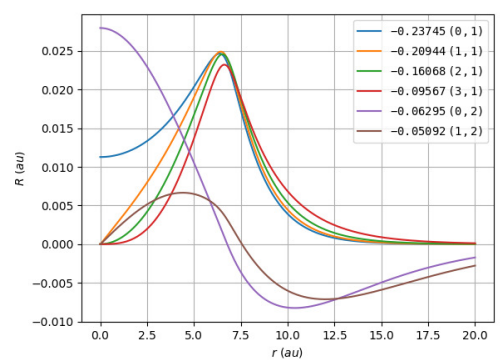

b)

Figure 5. Results The spectrum of charged fullerene for the potential $U(r)=U_{1}(r)+U_{2}(r)$ with $Z=1$ : a) the spectrum of charged fullerene, b) radial wave functions $R(r)$ for the lowest levels. 
Table 6. The energy levels in the potential $U(r)=U_{1}(r)+U_{2}(r)$ of a charged fullerene with $Z=5$

\begin{tabular}{cccccccc}
\hline$n$ & $l$ & $i$ & $E$ & $n$ & $l$ & $i$ & $E$ \\
\hline 1 & 0 & 1 & -0.81036 & 7 & 1 & 2 & -0.49728 \\
2 & 1 & 1 & -0.77750 & 8 & 5 & 1 & -0.44581 \\
3 & 2 & 1 & -0.72167 & 9 & 0 & 3 & -0.41576 \\
4 & 3 & 1 & -0.64593 & 10 & 2 & 2 & -0.41383 \\
5 & 0 & 2 & -0.60188 & 11 & 1 & 3 & -0.36906 \\
6 & 4 & 1 & -0.55291 & 12 & 3 & 2 & -0.35775 \\
\hline
\end{tabular}

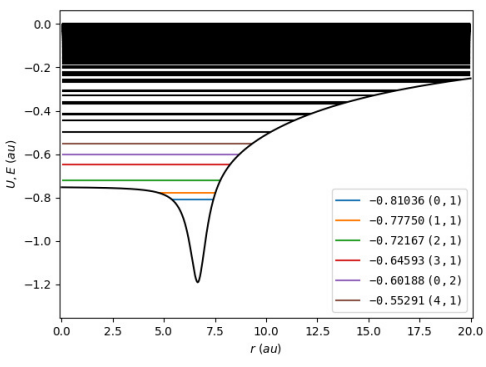

a)

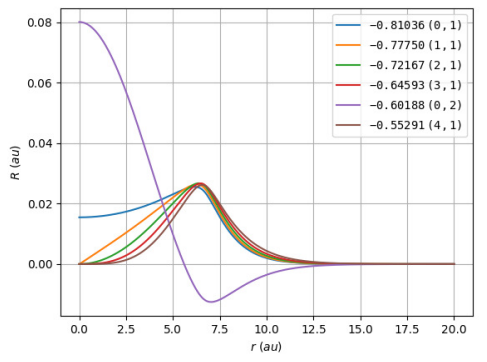

b)

Figure 6. Results for for the potential $U(r)=U_{1}(r)+U_{2}(r)$ of a charged fullerene with $Z=5$ : a) the spectrum, b) the radial wave function.

\section{Conclusions}

- A simple mathematical model for describing the single-electron groundstate spectrum of a charged fullerene is proposed. It is based on the use of the potential of a charged sphere and the spherically symmetric potential of an neutral fullerene for the Schrödinger equation.

- The computational algorithm for solution of the spectral problem is based on the use of piecewise linear finite elements and tested on the problem of the spectrum of the hydrogen atom. The software implementation is based on the free library for spectral problems solution SLEPc.

- Results of calculations demonstrate the existence of a system of discrete short-lifetime quantum states for electrons in the potential well of the self-consistent Coulomb field of charged fullerenes. Owing to its singleelectron nature, the current model can realistically access the low-energy excited states or capture-decays far below the plasmonic energies.

- For neutral fullerenes, attention is usually focused on distinctly edge effects at the position of the carbon atomic cage. A novel feature for charged fullerenes is presence of the volume-localized phenomena. A possible experimental confirmation of the existence of the volume-localized discrete levels would be of considerable interest for the experimental research and practical tasks, including the development of new sources of coherent radiation in a wide range of wavelengths. Preliminary estimates 
of the lifetimes of the levels and of the inverse population value can be found in $[2]$.

\section{References}

[1] M.Y. Amusia, A.S. Baltenkov and B.G. Krakov. Photodetachment of negative $C_{60}^{-}$ions. Physics Letters A, 243(1-2):99-105, 1998. https://doi.org/10.1016/S0375-9601(98)00158-3.

[2] R.V. Arutyunyan and A.V. Osadchy. The systems of volume-localized electron quantum levels of charged fullerenes. Journal of Nanomaterials, 2018:7526869, 2018. https://doi.org/10.1155/2018/7526869.

[3] R.V. Arutyunyan, P.N. Vabishchevich and Y.N. Obukhov. Existence of a system of discrete volume-localized quantum levels for charged fullerenes. Physical Review B, 98(10):155427, 2018. https://doi.org/10.1103/PhysRevB.98.155427.

[4] A.S. Baltenkov, S.T. Manson and A.Z. Msezane. Jellium model potentials for the $C_{60}$ molecule and the photoionization of endohedral atoms, $A @ C_{60}$. Journal of Physics B: Atomic, Molecular and Optical Physics, 48(18):185103, 2015. https://doi.org/10.1088/0953-4075/48/18/185103.

[5] A.K. Belyaev, A.S. Tiukanov, A.I. Toropkin, V.K. Ivanov, R.G. Polozkov and A.V. Solov'yov. Photoabsorption of the fullerene $C_{60}$ and its positive ions. Physica Scripta, 80(4):048121, 2009. https://doi.org/10.1088/00318949/80/04/048121.

[6] V.R. Bhardwaj, P.B. Corkum and D.M. Rayner. Internal laser-induced dipole force at work in $C_{60}$ molecule. Physical Review Letters, 91(20):203004, 2003. https://doi.org/10.1103/PhysRevLett.91.203004.

[7] A. Brenac, F. Chandezon, H. Lebius, A. Pesnelle, S. Tomita and B.A. Huber. Multifragmentation of highly charged $C_{60}$ ions: Charge states and fragment energies. Physica Scripta, 1999(T80B):195, 1999. https://doi.org/10.1238/Physica.Topical.080a00195.

[8] S.C. Brenner and L.R. Scott. The Mathematical Theory of Finite Element Methods. Springer, New York, 2008. ISBN 9780387759333. https://doi.org/10.1007/978-0-387-75934-0.

[9] E. Campbell. Fullerene collision reactions. Kluwer Academic, Dordrecht London, 2003. ISBN 9781402025242.

[10] J. P. Connerade, V.K. Dolmatov, P.A. Lakshmi and Manson S.T. Electron structure of endohedrally confined atoms: atomic hydrogen in an attractive shell. Journal of Physics B: Atomic, Molecular and Optical Physics, 32(10):L239L245, 1999. https://doi.org/10.1088/0953-4075/32/10/101.

[11] J.P. Connerade, V.K. Dolmatov and Manson S.T. A unique situation for an endohedral metallofullerene. Journal of Physics B: Atomic, Molecular and Optical Physics, 32(14):L395-L403, 1999. https://doi.org/10.1088/09534075/32/14/108.

[12] S. Díaz-Tendero, M. Alcamí and F. Martín. Structure and electronic properties of highly charged $C_{60}$ and $C_{58}$ fullerenes. The Journal of Chemical Physics, 123(18):184306, 2005. https://doi.org/10.1063/1.2104467.

[13] Z. Felfli and A.Z. Msezane. Simple method for determining binding energies of fullerene negative ions. European Physical Journal B, 72:78, 2018. https://doi.org/10.1140/epjd/e2018-80420-9. 
[14] V. Hernandez, J.E. Roman and V. Vidal. SLEPc: A scalable and flexible toolkit for the solution of eigenvalue problems. ACM Transactions on Mathematical Software (TOMS), 31(3):351-362, 2005. https://doi.org/10.1145/1089014.1089019.

[15] V.K. Ivanov, G.Y. Kashenock, R.G. Polozkov and A.V. Solov'yov. Photoionization cross sections of the fullerenes $C_{20}$ and $C_{60}$ calculated in a simple spherical model. Journal of Physics B: Atomic, Molecular and Optical Physics, 34(21):L669-L677, 2001. https://doi.org/10.1088/0953-4075/34/21/101.

[16] W. Jaskólski. Confined many-electron systems. Physics Reports, 271(1):1-66, 1996. https://doi.org/10.1016/0370-1573(95)00070-4.

[17] J. Jensen, H. Zettergren, H.T. Schmidt, H. Cederquist, S. Tomita, S.B. Nielsen, J. Rangama, P. Hvelplund, B. Manil and B. A. Huber. Ionization of $C_{70}$ and $C_{60}$ molecules by slow highly charged ions: A comparison. Physical Review A, 69:053203, 2004. https://doi.org/10.1103/PhysRevA.69.053203.

[18] W. Krätschmer, L.D. Lamb, K. Fostiropoulos and D. R. Huffman. Solid $C_{60}$ : a new form of carbon. Nature, 347(6291):354-358, 1990. https://doi.org/10.1038/347354a0.

[19] H.W. Kroto, J.R. Heath, S.C. O'Brien, R.F. Curl and R.E. Smalley. $\quad C_{60}: \quad$ Buckminsterfullerene. Nature, 318(6042):162-163, 1985. https://doi.org/10.1038/318162a0.

[20] L.D. Landau and E.M. Lifshitz. Quantum Mechanics : Non-Relativistic Theory. Elsevier Science, Burlington, 1977. ISBN 9781483149127.

[21] F. Langa. Fullerenes: principles and applications. Royal Society of Chemistry, Cambridge, U.K, 2011. ISBN 9781849732956.

[22] V. Ledoux and M. Van Daele. Matslise 2.0: A Matlab toolbox for SturmLiouville computations. ACM Transactions on Mathematical Software (TOMS), 42(4):29:1-29:18, June 2016. https://doi.org/10.1145/2839299.

[23] A. Logg, K. A. Mardal and G. Wells. Automated Solution of Differential Equations by the Finite Element Method: The FEniCS Book. Springer, Berlin New York, 2012. ISBN 9783642230981. https://doi.org/10.1007/978-3-642-23099-8.

[24] L.L. Lohr and M. Blinder. Electron photodetachment from a Dirac bubble potential. a model for the fullerene negative ion $C C_{60}^{-}$. Chemical Physics Letters, 198(1-2):100-108, 1992. https://doi.org/10.1016/0009-2614(92)90055-R.

[25] M.E. Madjet, H.S. Chakraborty, J.M. Rost and S.T. Manson. Photoionization of $C_{60}$ : a model study. Journal of Physics B: Atomic, Molecular and Optical Physics, 41(10):105101, 2008. https://doi.org/10.1088/09534075/41/10/105101.

[26] R.G. Polozkov, V.K. Ivanov and A.V. Solov'yov. Photoionization of the fullerene ion $C_{60}^{+}$. Journal of Physics B: Atomic, Molecular and Optical Physics, 38(24):4341-4348, 2005. https://doi.org/10.1088/0953-4075/38/24/001.

[27] J. Pryce. Numerical solution of Sturm-Liouville problems. Clarendon Press, Oxford England New York, 1993. ISBN 0198534159.

[28] J.D. Pryce. A test package for Sturm-Liouville solvers. ACM Transactions on Mathematical Software, 25(1):21-57, 1999. https://doi.org/10.1145/305658.287651.

[29] M.J. Puska and R.M. Nieminen. Photoabsorption of atoms inside $C_{60}$. Physical Review A, 47:1181, 1993. https://doi.org/10.1103/PhysRevA.47.1181. 
[30] A. Rüdel, R. Hentges, U. Becker, H.S. Chakraborty, M.E. Madjet and J.M. Rost. Imaging delocalized electron clouds: Photoionization of $C_{60}$ in Fourier reciprocal space. Physical Review Letters, 89:125503, 2002. https://doi.org/0.1103/PhysRevLett.89.125503.

[31] Y. Saad. Numerical methods for large eigenvalue problems. Society for Industrial and Applied Mathematics, Philadelphia, 2011. ISBN 9781611970722. https://doi.org/10.1137/1.9781611970739.

[32] R. Sahnoun, K. Nakai, Y. Sato, H. Kono, Y. Fujimura and M. Tanaka. Theoretical investigation of the stability of highly charged $C_{60}$ molecules produced with intense near-infrared laser pulses. The Journal of Chemical Physics, 125(18):184306, 2006. https://doi.org/10.1063/1.2371109.

[33] S. Saito, A. Oshiyama and Miyamoto Y. Electronic structures of fullerenes and fullerides. Computational Approaches in Condensed-Matter Physics, pp. 22-26, 1992. https://doi.org/10.1007/978-3-642-84821-6_4.

[34] G. Sánchez, S. Díaz-Tendero, M. Alcamí and F. Martín. Size dependence of ionization potentials and dissociation energies for neutral and singly-charged $C_{n}$ fullerenes $(n=40-70)$. Chemical Physics Letters, 416(1-3):14-17, 2005. https://doi.org/10.1016/j.cplett.2005.09.033.

[35] K. Sattler. Handbook of nanophysics. CRC Press, Boca Raton, 2011. ISBN 9781420075540 .

[36] G. Schrange-Kashenock. $4 d \rightarrow 4 f$ resonance in photoabsorption of cerium ion $\mathrm{Ce}^{3+}$ and endohedral cerium in fullerene complex $C e @ C_{82}^{+}$. Journal of Physics B: Atomic, Molecular and Optical Physics, 49(19):185002, 2016. https://doi.org/10.1088/0953-4075/49/18/185002.

[37] G.W. Stewart. A Krylov-Schur algorithm for large eigenproblems. SIAM Journal on Matrix Analysis and Applications, 23(3):601-614, 2001. https://doi.org/10.1137/S0895479800371529.

[38] N. Troullier and J.L. Martins. Structural and electronic properties of $C_{60} . \quad$ Physical Review B, 46(3):1754-1765, 1992. https://doi.org/10.1103/PhysRevB.46.1754.

[39] A.V. Verkhovtsev, A.V. Korol and Solov'yov A.V. Quantum and classical features of the photoionization spectrum of $C_{60}$. Physical Review A, 88:043201, 2013. https://doi.org/10.1103/PhysRevA.88.043201.

[40] Y.B. Xu, M.Q. Tan and U. Becker. Oscillations in the photoionization cross section of $C_{60}$. Physical Review Letters, 76:3538, 1996. https://doi.org/10.1103/PhysRevLett.76.3538.

[41] K. Yabana and G.F. Bertsch. Electronic structure of $C_{60}$ in a spherical basis. Physica Scripta, 48(5):633-637, 1993. https://doi.org/10.1088/0031$8949 / 48 / 5 / 022$.

[42] H. Zettergren, J. Jensen, H.T. Schmidt and H. Cederquist. Electrostatic model calculations of fission barriers for fullerene ions. European Physical Journal D: Atomic, Molecular, Optical and Plasma Physics, 29(1):63-68, 2004. https://doi.org/10.1140/epjd/e2004-00006-6.

[43] H. Zettergren, G. Sánchez, S. Díaz-Tendero, M. Alcamí and F. Martín. Theoretical study of the stability of multiply charged $C_{70}$ fullerenes. The Journal of Chemical Physics, 127(10):104308, 2007. https://doi.org/10.1063/1.2768361.

[44] A. Zettl. Sturm-Liouville theory. American Mathematical Society, Providence, R.I, 2005. ISBN 9780821839058. 